\title{
Quantitative Information from Cryo Electron Tomography of Energy Materials
}

\author{
Elliot Padgett ${ }^{1}$, Nina Andrejevic ${ }^{1}$, Zhongyi Liu ${ }^{2}$, Koji Moriyama ${ }^{3}$, Ratandeep Kukreja ${ }^{4}$, Swami \\ Kumaraguru $^{2}$, Wenbin Gu${ }^{4}$, Yi Jiang ${ }^{5}$, Veit Elser ${ }^{5}$, David A. Muller ${ }^{1}$. \\ 1. School of Applied and Engineering Physics, Cornell University, Ithaca, NY. \\ 2. Chemical and Materials Systems Laboratory, General Motors, Warren, MI. \\ 3. Honda R\&D Co. Ltd. Automobile R\&D Center, Haga-machi, Tochigi, Japan. \\ 4. GM Global Powertrain Engineering, Pontiac, MI. \\ 5. Department of Physics, Cornell University, Ithaca, NY.
}

STEM tomography has become a powerful tool for characterizing the 3D microstructure of materials at nanometer resolution. [1] To establish structure-performance relationships for energy materials, tomography must provide quantitative information that can be reliably compared between samples and benchmarked to bulk measurements. Here we use cryo-STEM tomography to study carbon-supported platinum fuel cell catalysts and make quantitative comparisons across support morphologies and catalyst loading. Our statistical measurements are in good agreement with bulk electrochemistry, but also provide structural insights needed for modeling, such as increased catalyst confinement at lower loading.

Carbon-supported platinum presents a good test case for tomography methods, combining nm-scale platinum nanoparticles and pores in weakly-scattering, dose-sensitive carbon. To suppress both carbon contamination and ice accumulation, each of which obscures the carbon support, we cool samples to $100^{\circ} \mathrm{C}$. Thermal drift (which is exacerbated in cryo tomography) introduces shear and compression distortions to STEM images, which degrade reconstruction resolution, creating streaks and false void artifacts. We prevent drift artifacts by acquiring sets of fast-scanned images to be aligned and summed in post-processing (Fig 1). Strong scattering from the platinum introduces artifacts in the carbon reconstruction and interferes with identification of carbon features. To suppress these artifacts, we image using low-angle annular dark field STEM to enhance the C/Pt signal ratio and use the simultaneous iterative reconstruction technique followed by threshold segmentation and morphological smoothing. For unloaded carbon, weighted back-projection allows straightforward segmentation of pore structure.

We measure the statistics of platinum particle size, surface area, and position on the carbon for $10 \mathrm{wt} \%$ and 50wt\% platinum loadings on Vulcan carbon black and high surface area carbon (HSC) (Fig 2). Total surface area measured by tomography is consistent with electrochemical surface area. We find that in HSC, which is relatively porous, the majority of the catalyst is embedded in the carbon interior, while in Vulcan the majority is on the carbon exterior. Increasing platinum loading results in much larger exterior particles and an increased fraction of surface area on the exterior, especially in Vulcan, as the confining interior pores appear to become saturated. In unloaded HSC we measure the distribution of pore and bottleneck sizes, finding that the majority of pore volume is accessible only through much smaller constrictions (Fig 3), supported by nitrogen sorption isotherms. Together, these results highlight the importance of understanding transport through interior micropores in pursuit of lower platinum fuel cells and provide a physical grounding for modeling efforts. [2]

References:

[1] PA Midgely and M Weyland, Ultramicroscopy 96 (2003), p. 413-431.

[2] Research funded by GM \& Honda. E Padgett acknowledges support from a NSF Graduate Research Fellowship (DGE-1144153). EM Facility support from the NSF MRSEC program (DMR 1120296). 

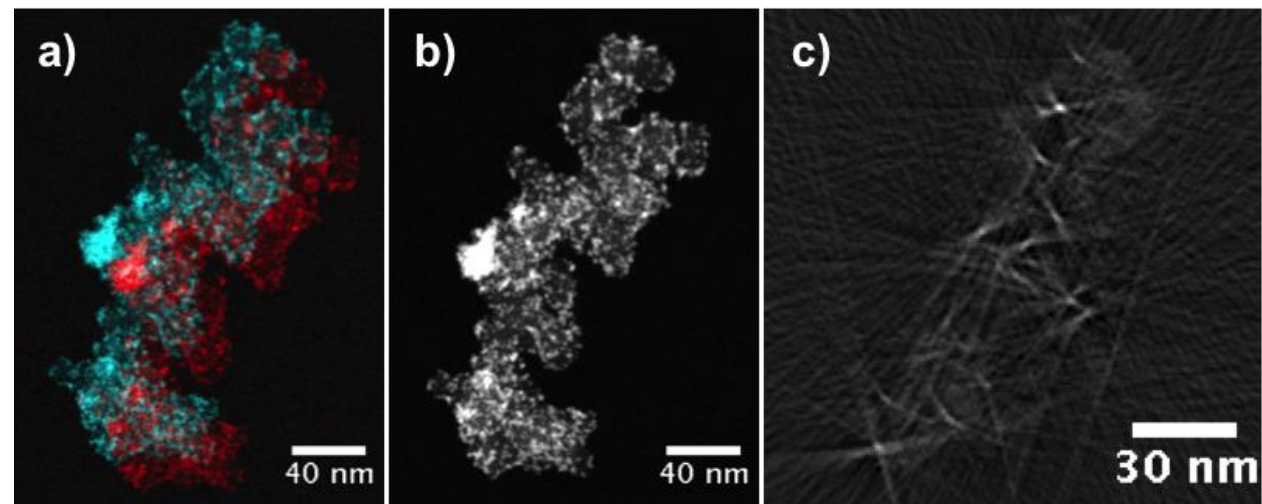

d)

Figure 1. a) Illustration of drift during cryo-STEM tomography with composite of images from beginning (blue) and end (red) of a 24 second acquisition and sum of cross-correlation-aligned 1 second images (b). Comparison of WBP reconstructions of cryo-tomography data taken with ordinary STEM imaging showing severe artifacts (c), and with fast-scan imaging clearly resolving nanoparticles (d).
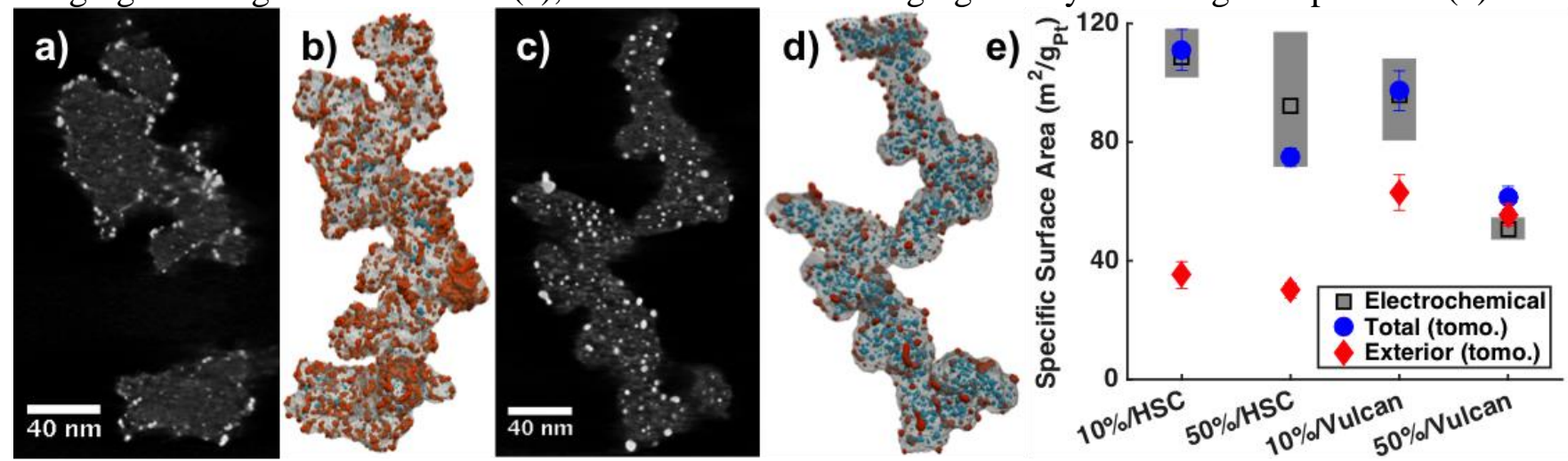

Figure 2. Cross sections (a,c) of SIRT reconstructions (square-root intensity to highlight carbon) and 3D surface renderings $(\mathrm{b}, \mathrm{d})$ of segmented reconstructions showing carbon (grey), exterior catalyst particles (red), and interior catalyst particles (blue) for $50 \mathrm{wt} \% \mathrm{Pt} /$ Vulcan and $50 \mathrm{wt} \% \mathrm{Pt} / \mathrm{HSC}$ respectively. e) Comparison of specific surface area measured by tomography for all catalyst particles (blue), exterior particles (red), and electrochemical surface area by hydrogen adsorption-desorption.

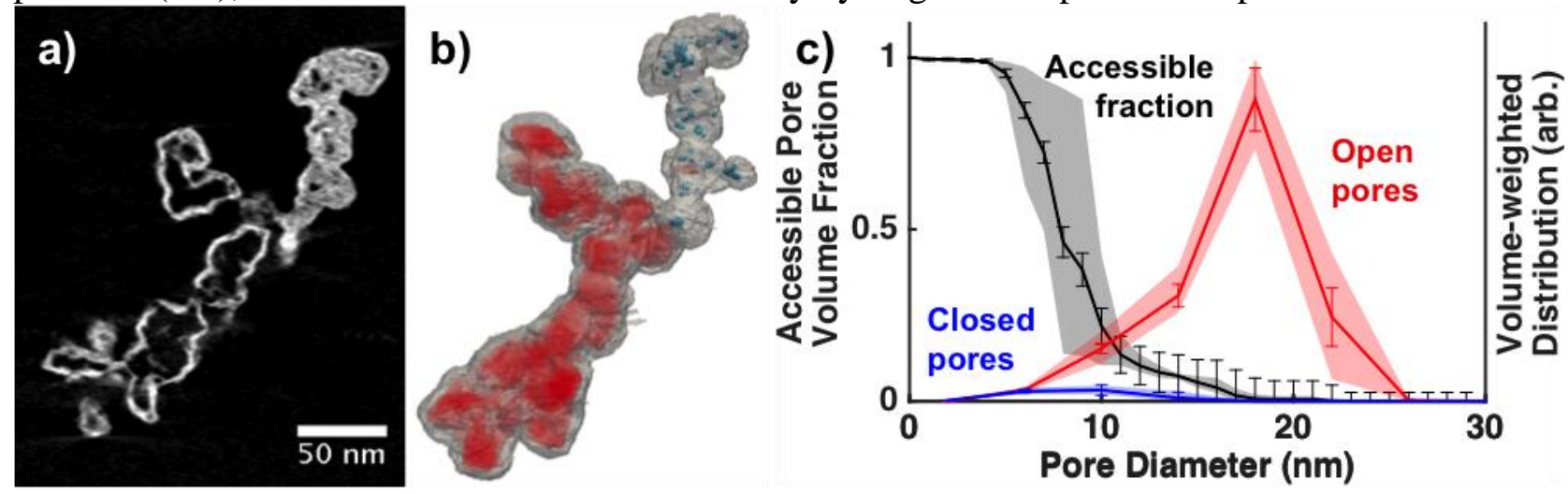

Figure 3. a) Cross section of WBP reconstruction of porous HSC carbon aggregate. b) 3D surface rendering of segmented reconstruction showing carbon (grey), closed pores (blue), and open pores (red). c) Plot of volume-weighted diameter distribution for open and closed pores with the fraction of pore volume accessible through pores of a given diameter or greater. Error bars show statistical uncertainty and shading shows segmentation uncertainty. 